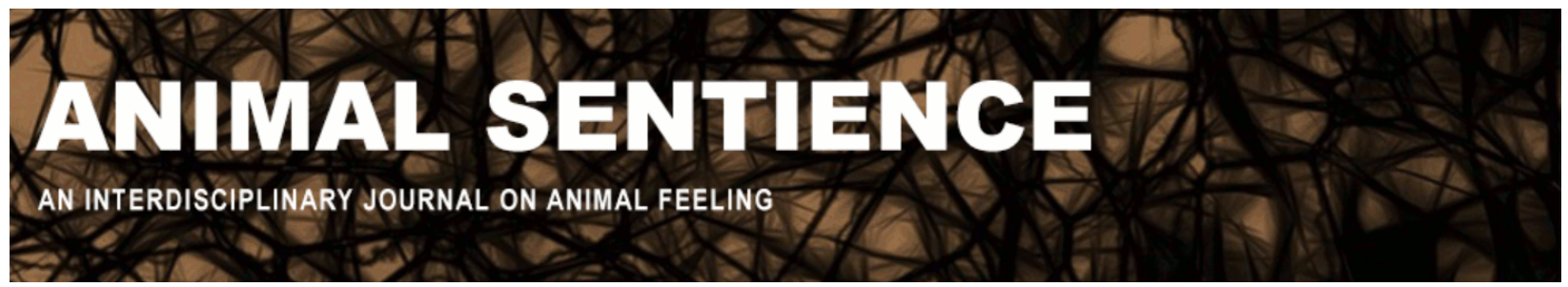

King, Barbara J. and Marino, Lori (2019) Octopus minds must lead to octopus ethics. Animal Sentience 26(14)

DOI: $10.51291 / 2377-7478.1495$

Date of submission: 2019-06-22

Date of acceptance: 2019-07-01 (c) 


\title{
Octopus minds must lead to octopus ethics
}

Commentary on Mather on Octopus Mind

\author{
Barbara J. King \\ College of William and Mary \\ Lori Marino \\ Kimmela Center for Animal Advocacy
}

\begin{abstract}
Mather argues convincingly for the existence of minds in octopuses based largely on laboratory experiments. Many of these experiments are highly invasive and involve mutilation and death. Moreover, octopuses are now being hailed as a "new model" for biological research and are being enthusiastically bred in captivity, both for research and for food. We argue that the compelling evidence for mind in octopuses must be accompanied by intense scrutiny of the ethics that shape how we treat them and that the intrinsic value of their individual lives must be recognized.
\end{abstract}

\begin{abstract}
Barbara J. King, Professor Emerita of Anthropology, College of William and Mary, writes and speaks about animal thinking, feeling, and ethics. Among her books are How Animals Grieve and Personalities on the Plate: The Lives \& Minds of Animals We Eat. Her work has appeared on NPR and in Scientific American, the Atlantic, Aeon, Undark, and TLS. Website
\end{abstract}

Lori Marino, neuroscientist, President, Whale Sanctuary Project, and Executive Director, Kimmela Center for Animal Advocacy, has written over 130 publications on dolphin and whale brain evolution and anatomy; intelligence and self-awareness; and the effects of captivity on social mammals, including cetaceans, elephants and primates. Website
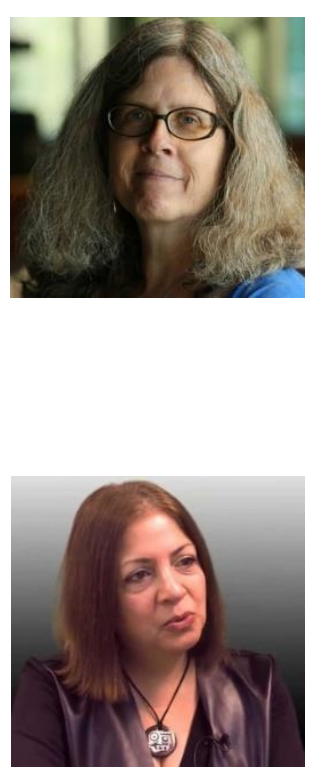

Jennifer Mather (2019), an expert on cephalopods, makes a compelling case that octopuses have minds. By "mind" she means that they reason about how to negotiate the world around them and respond flexibly rather than always relying on programmed or stereotyped responses. Octopuses may select specific arms for specific tasks, for example, and elect to coordinate those arms; they can anticipate a future need, as when they carry coconut shells a distance in the sea then assemble them into a shelter; and they use past information from foraging trips to guide their decision-making during new ones. The world of an octopus is driven, Mather notes, by curiosity, exploration, and also fear. 
In our commentary, we take up the implications of these features of octopus life for the ethical treatment of these animals. We discuss how the existence of the octopus mind could indeed should - inform discussions in two contexts: the breeding of octopuses for invasive scientific experiments and large-scale farming of octopus in ways poised to replicate the factory farms in which pigs, chickens, cows and other farmed animals are tortured and killed.

Mather argues that in order to fully understand how octopuses think, "we need both the control of laboratory experiments and the ecological validity of field observations." A sizeable part of what we know about octopus biology and learning comes from captive experiments over the last decades. Yet Mather follows up her statement by noting that captive testing may occur "in an ecologically inappropriate context" and goes on to say that "observations of octopuses in the laboratory are biased because the environment lacks adequate space." This is a mixed message, at best, about the validity and significance of captive studies.

Octopuses lack protection under the Animal Welfare Act or other animal-welfare legislation in the U.S. (whereas the EU has extended protection to cephalopods recently; Smith et al. 2013). Mather cites evidence that octopuses self-monitor their body state, noting that "when an arm is damaged," octopuses tend their wounds (Alupay, Hadjilosolomou, \& Crook, 2014). What she does not describe is the source of those wounds: crush injuries administered to five algae octopuses by the researchers in an experiment. First the researchers temporarily deactivated the animals' cerebrums, using anesthesia, and then they removed some of their arms. Later, once the animals were awake, they applied electrodes to the stumps or crush sites. The octopuses' response included holding the injured area against the body or curling other arms around the wound site. This wound-tending did not lead researchers to conclude that octopuses feel pain. Mather speaks of "pain" rather than pain in octopuses. Whatever we call it, a case for the octopus mind is a case against this type of experimentally induced crush injury or similarly harmful procedures: is it conceivable that animals who reason, plan, and remember, do not suffer from such treatment?

One of us (BJK) accepts the value of some studies on octopus behavior in captivity, specifically those designed for non-invasive observation of a few animals that would benefit species management in the wild, with safe release of the animals back into the wild at the end of the experimental period. LM thinks the welfare issues in captivity for octopuses are too onerous to justify even temporary non-invasive laboratory research in anything other than extreme cases of need to conserve free-ranging octopus populations or species.

A major concern is a new push for scaling up the use of cephalopods, including octopuses, as animal models in various kinds of invasive research. The Marine Biological Laboratory in Woods Hole, Massachusetts, currently houses about 3,000 individuals, including octopuses, in its threeyear-old Cephalopod Program (Greenfieldboyce, 2019). The program's purpose is to decode cephalopods' genetic sequences and to manipulate the genetic code to produce variants of normal animals. The program head, Joshua Rosenthal, described this to the Washington Post as "the first effort to go make a genetically tractable model" (Guarino, 2019). The question, of course, is: a model for what? In a six-minute video posted on the Marine Biological Laboratory's website, there is mention of microscopy, embryology, genetic work via injection, and work involving the regeneration of limbs. The lab manager, Bret Grasse, explains that theirs is probably the largest research collection of cephalopods in the world, and that it is "growing 
extremely rapidly," with a goal of making this "new resource" available to researchers who would benefit from investigating a different animal.

Grasse notes that it is possible to diagnose a "happy cephalopod" by checking for the presence of species-specific behaviors. At the same time, a viewer sees row upon row of small tanks, and might wonder, where is the opportunity for octopuses to use their mind? How could such animals be "happy" in such confined artificial conditions (leaving aside the procedures they are subjected to when removed from their tanks)? How does the commitment to breeding and shipping out more and more octopuses (and squid) to researchers fit with Mather's recognition of great capacities for exploration and thought in these animals? Ironically, most researchers who conduct studies of octopuses point to their large, complex and sophisticated brains as reasons for wanting to study them, completely overlooking the fact that this might be the very reason that should give us pause.

A parallel scaling-up is occurring with octopus farming. New initiatives for "ranching" octopuses globally are increasing at a rapid pace along with investment. Long considered a delicacy in many cultures around the world - and sometimes served live to consumers in a number of countries including the U.S. - octopus are seen as a good candidate for aquaculture because they grow rapidly, reproduce in quantity, and are appealing to consumers. Many of these facilities do not even provide a hiding place for octopuses, an important part of their environment that allows them to express natural territoriality (Yeoman, 2015). These more "efficient" captive environments force octopuses into increasingly unnatural and sterile conditions resembling the horrific conditions of concentrated animal feeding operations (CAFOs).

Jacquet et al. (2019) point out ethical objections to octopus farming that mesh well with Mather's discussion of the octopus mind. They write:

"Aquaculture depends on tightly controlled and monotonous environments, with constant ambient conditions, simplified and sterile enclosures, and rigid feeding schedules, aimed at supporting high stocking densities. Many octopus species appear to be largely asocial and show little tolerance of other individuals of the same species. Farming such species that, in addition, are carnivorous will almost inevitably require that individuals be kept isolated in small containers, with no scope for environmental enrichment and very poor overall well-being."

Just as the scientific world is beginning to fully appreciate the octopus mind, the future for octopuses looks bleak. In describing the high rate of predation that wild octopuses experience, Mather writes, "The intelligent octopus must hence always be on guard, all the time and against everyone." We find this sentence especially poignant in light of rising initiatives to conduct invasive research on octopuses and to breed them in large numbers for food. It is, in fact, the human species that the octopuses' mind need fear most of all. We hope that Mather's call for understanding the octopus mind will become a platform for intense scrutiny of how octopuses are treated and for the development of a kinder approach to recognizing the intrinsic value of their lives. 


\section{References}

Alupay, J. A., Hadjisolomou, S. P., \& Crook, R. J. (2014). Arm injury produces long-term behavioral and neural hypersensitivity in octopus. Neuroscience Letters, 558, 137-142.

Greenfieldboyce, N. (2019). Why octopuses might be the next lab rats. National Public Radio. June 3.

Guarino, B. (2019). Inside the grand and sometimes slimy plan to turn octopuses into lab animals. Washington Post. March 2.

Jacquet, J., Franks, B., Godfrey-Smith, P., \& Sánchez-Suárez, W. (2019). The case against octopus farming. Issues in Science and Technology, 35(2), 37-44.

Mather, J. (2019). What is in an octopus's mind? Animal Sentience 26(1).

Smith, J. A., Andrews, P. L., Hawkins, P., Louhimies, S., Ponte, G., \& Dickel, L. (2013). Cephalopod research and EU Directive 2010/63/EU: Requirements, impacts and ethical review. Journal of Experimental Marine Biology and Ecology, 447, 31-45.

Yeoman, R. (2015). Brave new world of octopus farming. Fish 23(1). 